\title{
Vertebral haemangioma compared to bony metastasis on magnetic
} resonance imaging

\author{
Scott F Farrell ${ }^{1,2}$ \\ Peter G Osmotherly ${ }^{2}$ \\ Peter $\mathrm{Lau}^{3}$
}

1. Postdoctoral Fellow, RECOVER Injury Research Centre, School of Allied Health

Sciences, Griffith University, Gold Coast, Queensland, Australia

2. Senior Lecturer, Discipline of Physiotherapy, School of Health Sciences, The University of Newcastle, Newcastle, New South Wales, Australia

3. Senior Staff Specialist Radiologist and Pain Management Clinician, Hunter New England Imaging and Department of Clinical Research and Pain Management, Newcastle, New South Wales, Australia

\section{Corresponding Author:}

Scott F Farrell

RECOVER Injury Research Centre

Building G05 Room 3.20

Griffith University Gold Coast Campus

Southport QLD 4222

Australia

Phone: +61 400550548

Fax: +61 755528674

Email: s.farrell@griffith.edu.au 


\section{Co-author Contact Details:}

Dr. Peter Osmotherly

School of Health Sciences

Hunter Building

The University of Newcastle

Callaghan NSW 2308

Australia

E: peter.osmotherly@newcastle.edu.au P: +612 49217718 F: +612 49217902

Dr. Peter Lau

Hunter New England Imaging

Locked Bag 1 HRMC

Newcastle NSW 2310

Australia

E: peter.lau@hnehealth.nsw.gov.au P: +612 49213434 F: +612 49213428

Institutional Ethics Review: Not applicable, de-identified imaging case report

Financial Disclosure Statement: No financial affiliation or involvement exists between the authors and any commercial organisation that has a direct financial interest in any matter included in this manuscript.

Funding details: Not applicable.

Conflicts of Interest: None to declare.

Key words: magnetic resonance imaging; thoracic spine; hemangioma; neoplasm metastasis; back pain; incidental findings. 


\section{Main Text}

Vertebral haemangiomas (VHs) are benign vascular lesions of vertebral bodies, and are common incidental findings in imaging, occurring in approximately $10-12 \%$ of the adult population. ${ }^{1}$ These lesions consist of thin-walled blood vessels that pervade the medullary cavity between trabecula. ${ }^{2}$ Typically, VHs are asymptomatic and do not require intervention unless causing neurological compromise. ${ }^{1}$

Health practitioners concerned with spinal pain, such as doctors or physiotherapists, often manage patients that have undergone magnetic resonance imaging (MRI). As such, it is likely that clinicians will encounter VHs when reviewing scans and reports. It is important therefore to understand this typically incidental finding to inform clinical reasoning, as well as for patient education and reassurance. The purpose of this report is to contrast the radiological appearance of $\mathrm{VH}$ with that of a sinister pathology requiring urgent medical attention - bony metastatic disease.

The case presented is a 67-year-old female with a background of breast cancer. She was referred for whole spine MRI due to increasing back pain. Imaging reveals both VH and metastatic disease of the thoracic spine.

VHs are hyperintense on both T1- and T2-weighted sequences due to their fat and water content, ${ }^{2}$ as can be seen at T7 (Figure 1). In contrast, bony metastatic disease is hypointense on T1-weighted sequences, and frequently (but not exclusively) hyperintense on T2-weighted sequences. ${ }^{3}$ This can be seen at T12 in Figure 1. This patient underwent treatment for spinal metastatic disease, and no management was required for the $\mathrm{VH}$. 
In summary, clinicians should be aware of VHs as common incidental findings on MRI.

These lesions have radiological characteristics distinct to bony metastatic disease, however if concerned about a scan, always defer to the expertise of our radiologist colleagues. ${ }^{4}$ 


\section{Figure:}

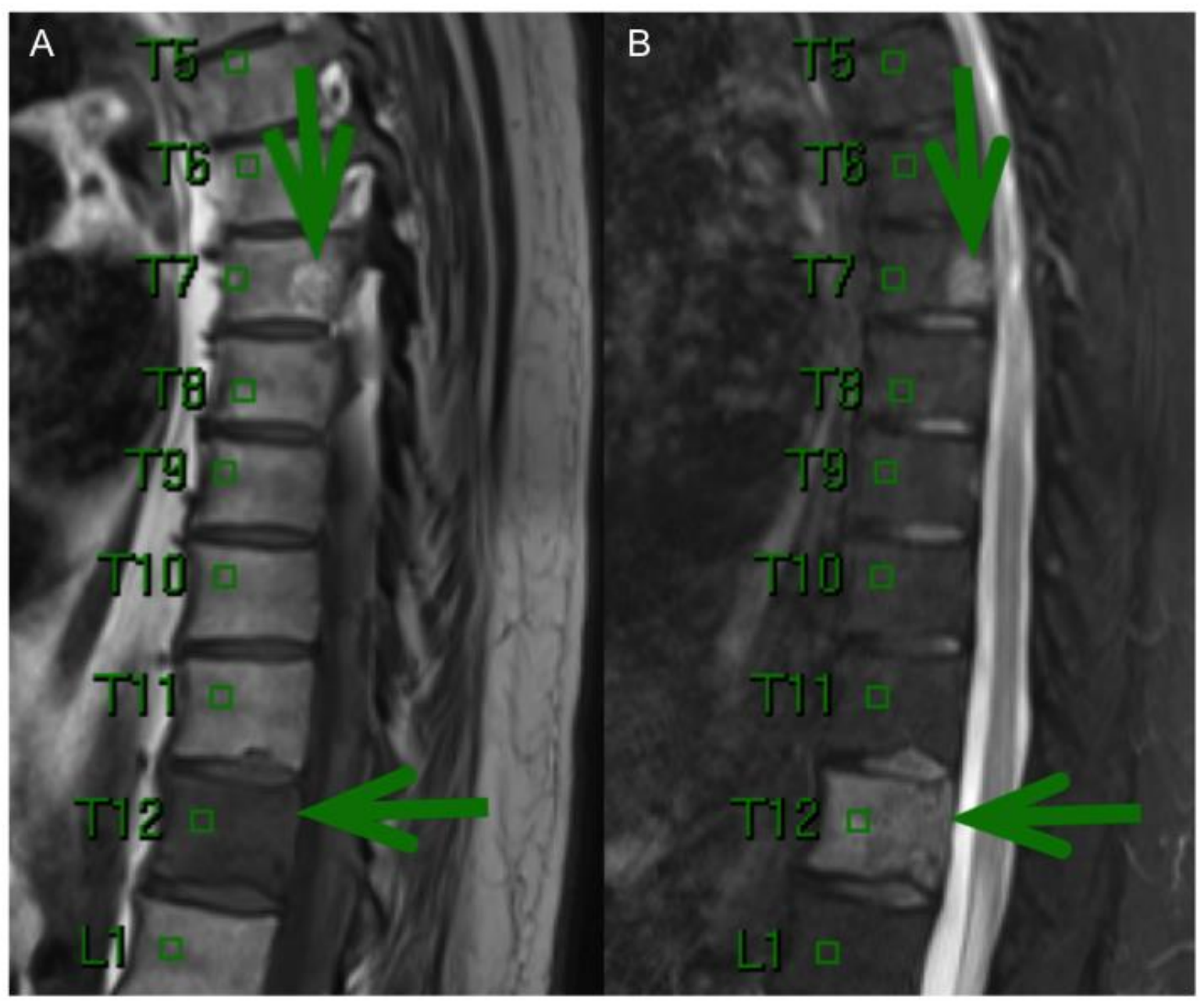

Figure 1: Sagittal magnetic resonance imaging of thoracic spine: a) T1-weighted sequence;

b) T2-weighted sequence. There is a vertebral haemangioma at T7: hyperintense on both T1and T2-weighted sequences. There is metastatic disease of the T12 vertebral body: hypointense on T1-weighted sequence, hyperintense on T2-weighted sequence. 


\section{References}

1. Alexander J, Meir A, Vrodos N, Yau YH. Vertebral hemangioma: An important differential in the evaluation of locally aggressive spinal lesions. Spine 2010;35:E917-E920.

2. Rodallec MH, Feydy A, Larousserie F, et al. Diagnostic imaging of solitary tumors of the spine: what to do and say. RadioGraphics 2008;28:1019-1041.

3. Guillevin R, Vallee JN, Lafitte F, Menuel C, Duverneuil NM, Chiras J. Spine metastasis imaging: Review of the literature. Journal of Neuroradiology 2007;34:311-321.

4. Elliott JM, Gorman I. Coincidental findings of a vertebral hemangioma on magnetic resonance imaging. Journal of Orthopaedic and Sports Physical Therapy 2009;39:149. 